\title{
A note on SINGLE-ITERATION SOBOLEV DESCENT FOR LINEAR INITIAL VALUE PROBLEMS
}

\author{
Sultan Sial ${ }^{1}$, Nauman Raza ${ }^{2}$, Adnan $\operatorname{Khan}^{1}$, and ahmad javid ${ }^{2}$ \\ ${ }^{1}$ Lahore University of Management Sciences Syed Babar Ali School of Science and \\ Engineering \\ ${ }^{2}$ University of the Punjab
}

April 28, 2020

\begin{abstract}
Mahavier and Montgomery construct a Sobolev space for approximate solution of linear initial value problems in a finite difference setting in SINGLE-ITERATION SOBOLEV DESCENT FOR LINEAR INITIAL VALUE PROBLEMS, Mahavier, Montgomery, MJMS, 2013. Their Sobolev space is constructed so that gradient-descent converges to a solution in a single iteration, demonstrating the existence of a best Sobolev gradient for finite difference approximation of solutions of linear initial value problems. They then ask if there is a broader class of problems for which convergence in a single iteration in an appropriate Sobolev space occurs. We use their results to show the existence of single-step iteration to solution in a lower dimensional Sobolev space for their examples and then a class of problems for single-step convergence.
\end{abstract}

\section{Hosted file}

single_Step_iteration.pdf available at https://authorea.com/users/306612/articles/437591-a-noteon-single-iteration-sobolev-descent-for-linear-initial-value-problems 
figures/Fig1/Fig1-eps-converted-to.pdf 
figures/Fig2/Fig2-eps-converted-to.pdf 
figures/fig3/fig3-eps-converted-to.pdf 
figures/fig4/fig4-eps-converted-to.pdf 